\title{
Empatía en niños de 11 a 12 años. Una comparación entre pedagogía Waldorf y pedagogía tradicional
}

\section{Empathy in children of 11 to 12 years. A Comparison Between Waldorf Pedagogy and Traditional Pedagogy}

* Carolina Esturao
cesturao@gmail.com

\author{
$* *$ Lucas G. Gago Galvagno \\ lucas.gagogalvagno@hotmail.com
}

\author{
**** Angel M. Elgier \\ amelgier@gmail.com
}

* Universidad Abierta Interamericana

** Universidad Abierta Interamericana - Universidad de Buenos Aires - CONICET

*** Universidad Abierta Interamericana - Universidad de Buenos Aires - CONICET

\section{Resumen}

$\mathrm{P}$ ara Eisenberg (2000) la empatía es una respuesta emocional que procede de la comprensión del estado o situación de otra persona y es similar a lo que la otra persona está sintiendo. La misma incluye la capacidad para diferenciar entre los estados afectivos de los demás y la habilidad para tomar una perspectiva tanto cognitiva como afectiva. Esta se ve modificada por procesos de socialización. La presente investigación tuvo como objetivo evaluar los niveles de empatía en niños de 11 y 12 años de edad en función de su asistencia a instituciones educativas bajo el modelo de la pedagogía tradicional o la pedagogía Waldorf, a partir del Interpersonal Reactivity Index (IRI). Los resultados indicaron que los alumnos que asistían al establecimiento Waldorf puntuaron más alto en toma de perspectiva y preocupación empática que los alumnos que asistían al establecimiento
Abstract

7 or Eisenberg (2000) empathy is an emotional response that comes from understanding the status or situation of another person and is similar to what the other person is feeling. It includes the ability to differentiate between the emotional states of others, and the ability to make a cognitive and affective perspective. This is modified by processes of socialization. This study aimed to assess levels of empathy in children 11 and 12 years depending on their attendance at educational institutions under the model of traditional pedagogy and Waldorf education, from the Interpersonal Reactivity Index (IRI). The results indicated that students attending the establishment Waldorf scored higher on perspective taking and empathic concern that students attending the establishment 


\section{Artículos Empíricos}

bajo el modelo de pedagogía tradicional. Se puede concluir que las diferencias encontradas entre los alumnos de las distintas escuelas podrían deberse a los distintos modelos pedagógicos que recibieron. La escuela Waldorf hace énfasis en estimular la competencia social, por lo que esto pudo repercutir en las habilidades empáticas de los niños.

Palabras clave: Empatía - pedagogía tradicional - pedagogía Waldorf. under the traditional model of pedagogy. It can be concluded that the differences among students of different schools could be due to different educational models they received. The Waldorf school emphasizes stimulating social competence, so this might affect the empathic abilities of children.

Keywords: Empathy - Traditional Pedagogy Waldorf Pedagogy.

\section{Introducción}

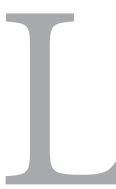

uego de la familia, la escuela es el medio fundamental que provee al niño de las herramientas necesarias para su desarrollo psicofísico (Acevedo \& Carrillo, 2010). Con tal propósito cada método educativo contempla como integrantes del proceso educativo a los alumnos y a los profesores, a su vez que considera el contexto institucional, el contenido y la tecnología. La diferencia con respecto a cada método se establece a partir de las distintas propuestas pedagógicas (Acevedo \& Carrillo, 2010).

Con respecto al método tradicional de la pedagogía, Palacios (1985) señala que la escuela tradicional significa, ante todo, método y orden. El fundamento de la pedagogía tradicional es el orden. La escuela tradicional basa su metodología de enseñanza en un modelo heteroestructurante. Dicho modelo concibe el saber como una construcción externa al salón de clases, donde la educación es un proceso de asimilación desde el exterior basado en la repetición y la copia (Palacios, 2010). Los métodos de enseñanza de la pedagogía tradicional se basan en la exposición verbal de la materia y/o la demostración. Los objetivos se elaboran de forma descriptiva, designados a la tarea del profesor, no a las acciones o habilidades a desarrollar por los alumnos. El énfasis es puesto en los ejercicios, en la repetición de conceptos expuestos o fórmulas, apuntando a disciplinar la mente y formar hábitos. El conocimiento se selecciona de modo empírico, por lo que hay un desarrollo del pensamiento teórico del estudiante; asimismo se le exige la memorización de los conocimientos expresados por el profesor, siendo en ocasiones ajenos a las experiencias vivenciales de los estudiantes (Navarro, 2010).

En este caso, el rol del maestro se presenta como base y condición del éxito educativo. Es el encargado de organizar, transmitir y elaborar el conocimiento, a fin de que este sea aprendido por el alumno. El maestro se propone como modelo, es quien marca el camino a seguir. En la dinámica diaria es escaso el uso de materiales sensoriales y el alumno dispone de un tiempo limitado para la realización de sus tareas (Acevedo \& Carrillo, 2010). 


\section{Artículos Empíricos}

Por otro lado, la pedagogía Waldorf es un método creado por el filósofo Rudolf Steiner que se constituyó sobre los principios rectores de la Antroposofía. El fundamento principal de dicha pedagogía, reside en una concepción particular del hombre, la de acompañarlo, desde niño, en sus diferentes etapas evolutivas mediante una educación integral respetando su individualidad y conducirlo de esta forma a ser un hombre libre y autónomo (Golderos, 2003). El objetivo de la educación Waldorf es lograr el desarrollo de seres humanos saludables en todos sus niveles, responsables y libres, considerando a la libertad como la facultad de decisión en cuanto a la acción, a la expresión y al pensamiento, tomando en cuenta el reconocimiento y respeto a los demás (Petrash, 2009).

La misma basa su didáctica en la creatividad del maestro, fundamentada en un seguimiento permanente del proceso evolutivo de cada niño y del grupo en su totalidad. La premisa principal de dicha pedagogía es respetar el proceso madurativo de cada niño, de forma equilibrada, fomentando sus capacidades intelectuales, su sensibilidad artística, su fuerza de voluntad y su autonomía; logrando una formación que en la adultez le permita responder a los desafíos de la vida, aportando nuevas ideas y proyectos a la sociedad. Es una metodología activa y creativa, el maestro produce nuevas estrategias de trabajo y didáctica según las necesidades de cada niño y de su clase en general (Steiner, 1985). En este caso, el rol del maestro es de acompañante y guía. El maestro permanece con un mismo grupo durante un período comprendido entre los seis años y la pubertad. Se establece así un vínculo personal que promueve continuidad, seguridad y que convierte al maestro en una autoridad natural.
El método Waldorf se centra en las necesidades del niño, se utiliza una amplia variedad de materiales sensoriales y cada niño toma su tiempo para aprender (Santillán, 2006). Un estudio detallado de los graduados de escuelas Waldorf (Barz \& Randoll, 2007) concluyen que los graduados en primer lugar encontraron más dificultades para orientarse en el mundo académico superior. Sin embargo, una vez que habían hecho la transición, estaban en mejores condiciones que el resto a desenvolverse en el curso y perseguir objetivos con éxito.

Por otro lado, Eisenberg (2000) definió la empatía como una respuesta emocional que surge de la comprensión del estado o situación de otra persona y que se asemeja a lo que la otra persona está sintiendo. La respuesta empática tiene que ver con la capacidad que tiene una persona de comprender al otro y de "ponerse en su lugar". El acceso a ese registro se obtiene a partir de lo observado, de la información verbal, de la información memorística y también de la reacción afectiva, al compartir su estado emocional, que puede causar tristeza, malestar o ansiedad. En esta línea, las interacciones con los pares y los maestros en un ámbito educativo específico podrían influenciar las características de este proceso.

La empatía ha llegado a un enfoque integrador que consideró los aspectos cognitivos y emocionales de forma conjunta. La misma fue denominada como una emoción positiva, por lo tanto, su aspecto cognitivo responde más a una inteligencia de carácter emocional que a un verdadero razonamiento cognitivo (Richaud, 2008). Richaud, (2008) investigó los índices de empatía en niños de 9 a 12 años de edad, que 


\section{Artículos Empíricos}

concurrían a escuelas primarias de la ciudad de Buenos Aires. En los resultados reportó que la empatía predice la conducta social en la niñez media y se observó que las personas empáticas son menos agresivas por su sensibilidad emocional y su capacidad para comprender las consecuencias negativas potenciales. En las últimas décadas se ha destacado la importancia de la empatía en la conducta social positiva, es decir prosocial y como ésta inhibe la agresividad (Mestre, Frías, \& Samper, 2004).

Para mensurar la empatía teniendo en cuenta sus distintas dimensiones, Davis desarrolló el Interpersonal Reactivity Index (IRI) que permite evaluar la empatía a través de cuatro factores: dos cognitivos y dos emocionales (Mestre, Frías, \& Samper, 2004).

A partir de 1980 se comenzó a trabajar en una definición integradora de la empatía, tanto con sus componentes afectivos como cognitivos. Así, Davis (1980) propuso una definición multidimensional de la empatía, que incluye tanto componentes cognitivos como emocionales. Estableció que se trata de un constructo que contiene cuatro elementos diferentes y relacionados entre sí. Dentro de la dimensión cognitiva distingue; Fantasía, que se refiere a la tendencia a identificarse con personas de ficción y Toma de perspectiva, como la capacidad de representarse la visión, los pensamientos y motivos que tiene el otro. Mientras que la dimensión emocional diferencia; Preocupación empática y el Distrés personal que miden las respuestas emocionales de las personas frente a las experiencias negativas de los otros (Mestre, Frías, \& Samper, 2004).
Surge entonces como interrogante si los procedimientos y estrategias propios de cada método, favorecen diferencias en las habilidades empáticas de cada niño.

La presente investigación se propone evaluar específicamente si hay diferencias en los puntajes de empatía en niños de 11 y 12 años de edad en función de su asistencia a Instituciones educativas bajo el modelo de la pedagogía tradicional o la pedagogía Waldorf, cuestión que presenta una falta de investigaciones.

\section{Método}

Se planteó un estudio cuantitativo, con alcance descriptivo comparativo, con un diseño no experimental, transaccional y para el cual se realizó un muestreo no probabilístico.

Con tal fin se recurrió a grupos “intactos” que cumplen los criterios de edad, sexo y pertenencia a un grupo escolar (tradicional o waldorf) establecidos para esta investigación. Los puntajes obtenidos permiten establecer comparaciones entre los niveles alcanzados por alumnos de escuela Tradicional y de escuela Waldorf.

\section{Participantes:}

Participaron de la muestra 60 sujetos de 11 a 12 años de edad, todos ellos alumnos de escuela primaria, de los cuales 30 asistían a escuela Tradicional y 30 a escuela Waldorf. El grupo que asistía a escuela Tradicional (Instituto San Vicente de Paul) estuvo conformado por 17 niñas y 13 niños. Y el grupo que asistía a escuela Waldorf (Escuela Juana de Arco) estuvo conformado por 20 niñas y 10 niños. Todos son residentes de la ciudad de Buenos Aires. 


\section{Artículos Empíricos}

Se realizó un muestreo simple intencional. Los criterios de inclusión fueron que los niños tuvieran entre 11 y 12 años, que hayan realizado su etapa de escolarización inicial y primaria una escuela con pedagogía Waldorf y tradicional, y que además asistan actualmente a ella. También que habiten en la Ciudad Autónoma de Buenos Aires y que tengan el consentimiento y autorización se sus padres para realización de los test.

\section{Materiales:}

Se utilizó el Índice de Reactividad Interpersonal (IRI) (Davis, 1980). Se trata de una escala de fácil aplicación, formada por 28 ítems distribuidos en cuatro subescalas que miden cuatro dimensiones del concepto global de la empatía: Toma de perspectiva (PT), Fantasía (FS), Preocupación empática (EC) y Distrés personal (PD), con siete ítems cada una de ellas.

Este es uno de los cuestionarios más utilizados para evaluar este constructo desde una perspectiva multidimensional, ya que incluye dos factores cognitivos y dos emocionales. Este instrumento permite medir tanto el aspecto cognitivo como la reacción emocional del individuo al adoptar una actitud empática; las escalas PT y FS evalúan los procesos cognitivos, la puntuación en Toma de perspectiva (PT) indica los intentos espontáneos del sujeto por adoptar la perspectiva del otro ante situaciones reales de la vida cotidiana, es decir, la habilidad para comprender el punto de vista de la otra persona. La subescala Fantasía (FS) la capacidad imaginativa del sujeto para ponerse situaciones ficticias (Mestre, Frias, \& Samper, 2004).
Mestre, Frias, \& Samper (2004) investigaron la medida de la empatía a través del Interpersonal Reactivity Index (IRI). El objetivo de dicha investigación fue analizar las propiedades psicométricas del IRI en su adaptación española. La muestra tomada fue de 1.285 adolescentes, 698 varones y 597 mujeres, con un rango de edad entre 13 y 18 años, pertenecientes a diversos centros educativos de la Comunidad Valenciana. Los resultados obtenidos indican que el instrumento es válido para evaluar los diferentes componentes de la empatía.

Las subescalas de Preocupación empática (EC) y Distrés personal (PD) evalúan los procesos emocionales de las personas frente a las experiencias negativas de los otros. En la primera (EC) se miden los sentimientos orientados hacia otros, como compasión, preocupación y afecto ante el malestar de otras personas; en la segunda (PD) se miden los sentimientos orientados hacia el yo, como la ansiedad y el malestar que el sujeto manifiesta al observar las experiencias negativas de los demás (Mestre, Frias, \& Samper, 2004).

El formato de respuesta es en escala tipo likert, con cinco opciones de respuesta (de 1 a 5 ), de acuerdo al grado en que dicha afirmación lo represente $(1=$ no me describe bien; $2=$ me describe un poco; $3=$ me describe bastante bien; $4=$ me describe bien y $5=$ me describe muy bien).

\section{Procedimiento}

Se presentó a las instituciones seleccionadas, tanto Tradicional como Waldorf, el proyecto de investigación con el fin de que aprobaran la aplicación del instrumento psicométrico 


\section{Artículos Empíricos}

a sus alumnos de 11 y 12 años de edad. Se recurrió a escuelas primarias privadas, bajo la consideración de que las escuelas Waldorf son particulares, y el cumplimiento de dicho requisito garantizaría condiciones socioeconómicas análogas entre los sujetos. Las consideraciones éticas que se aplicaron fueron: información detallada a las escuelas sobre los propósitos de la investigación, naturaleza y contenido del test a aplicarse con el fin de obtener su consentimiento. Los padres fueron notificados con igual detalle mediante notas informativas, donde se solicitó por escrito, su consentimiento y autorización correspondientes para la participación de cada niño. En dicha nota fue explicitado que se mantendría la confidencialidad de los datos proporcionados por los alumnos, tanto de su identidad como de sus respuestas. También a los niños se les explicó que su participación era voluntaria y que la información que proporcionaran sería tratada en forma confidencial.

A los alumnos de cada escuela que aceptaron colaborar les fue aplicado colectivamente el Índice de Reactividad Interpersonal (IRI), instrumento psicométrico que fue descrito en el punto anterior. Se les indicó a los niños que debían responder distintas afirmaciones relacionadas con sus sentimientos y pensamientos según las diversas situaciones que se le presentaban en el cuestionario.

En todos los casos, el contexto determinado para esta fase del proyecto fue el salón de clases, donde una o dos maestras estuvieron presentes durante el desarrollo del test.

Análisis de datos: Los datos fueron procesados y analizados con el programa estadístico SPSS versión 21 para Windows.

Para comparar los puntajes de los grupos Tradicional y Waldorf en las distintas variables dependientes, se utilizó la prueba t de Student para muestras independientes. El alfa utilizado fue de $5 \%$.

\section{Resultados}

En la Tabla 1 se encuentran las medias y desvíos para cada grupo (Waldorf y Tradicional), en cada una de las cuatro subescalas del IRI: Toma de Perspectiva, Fantasía, Preocupación Empática y Distrés Personal.

Las cuatro subescalas tuvieron distribución normal, de acuerdo al análisis con KolmogorovSmirnov.

Tomando un alfa del 5\%, se hallaron diferencias significativas en las variables Toma de Perspectiva y Preocupación Empática. En el primer caso, la prueba de $t$ de muestras independientes arrojó los siguientes resultados: $t(58)=6.47, p<0.01$. En el segundo caso, los valores fueron $\mathrm{t}(58)=4.27, \mathrm{p}<0.01$. Es decir que los alumnos del grupo Waldorf puntuaron significativamente más en estas subescalas que los alumnos del grupo Tradicional.

No hubo diferencias significativas en la subescala Fantasía ni en Distrés Personal. 
Tabla 1

Media y Desvío estándar halladas en los grupos Waldorf y Tradicional para cada subescala.

\begin{tabular}{lllll}
\hline & Grupo & N & Media & $\begin{array}{l}\text { Desvío } \\
\text { estándar }\end{array}$ \\
\hline Toma de Perspectiva & Waldorf & 30 & 25,3667 & 4,68662 \\
& Tradicional & 30 & 18,7000 & 3,14204 \\
Fantasía & Waldorf & 30 & 19,8000 & 5,45451 \\
& Tradicional & 30 & 19,2667 & 4,25022 \\
Preocupación Empática & Waldorf & 30 & 25,7000 & 3,31298 \\
& Tradicional & 30 & 21,5000 & 4,24061 \\
Distrés Personal & Waldorf & 30 & 16,9667 & 3,32683 \\
& Tradicional & 30 & 18,8667 & 4,17491 \\
\hline
\end{tabular}

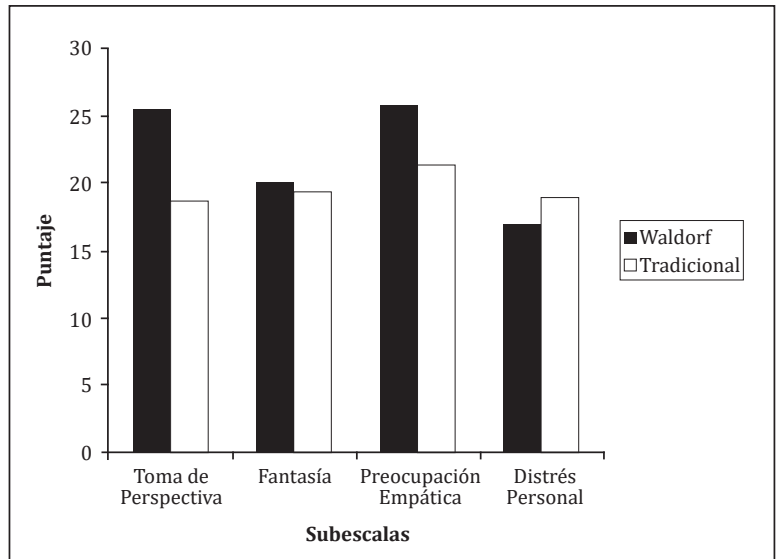

Figura 1. Media de ensayos en cada subescala del Interpersonal Reactivity Index (IRI). El análisis de datos fue realizado con una t de Student. Se hallaron diferencias significativas entre los grupos Waldorf y Tradicional para las subescalas "Toma de Perspectiva" y "Preocupación Empática".

\section{Discusión}

En este trabajo se buscó comparar el nivel de empatía de alumnos que asistían a un establecimiento educativo bajo la pedagogía Waldorf (entendida esta como una pedagogía participativa), y bajo la pedagogía tradicional (entendida esta como el modelo hegemónico occidental).

Hay escasos estudios empíricos sobre las consecuencias cognitivas y emocionales de asistir 


\section{Artículos Empíricos}

a un establecimiento bajo el modelo pedagógico Waldorf. En este sentido, otro de los objetivos fue estudiar de un modo sistemático los diversos efectos de la pedagogía Waldorf sobre un proceso cognitivo específico: la empatía.

Para esto se evaluaron 60 alumnos con la escala IRI, obteniendo diferencias significativas en la subescala Toma de Perspectiva y Preocupación Empática, puntuando más alto los niños que asistían a la escuela Waldorf en relación a los alumnos que asistían a la escuela bajo la pedagogía tradicional.

La toma de perspectiva indica los intentos espontáneos del sujeto por adoptar la perspectiva del otro ante situaciones reales de la vida cotidiana, es decir, la habilidad para comprender el punto de vista de la otra persona. Por otro lado, la preocupación empática evalúa los sentimientos de preocupación, compasión y cariño frente al malestar de otro. Es decir, que tipo de respuestas emocionales surgen ante las experiencias negativas de los otros (Mestre, Frías, \& Samper, 2004).

Damasio (1996) investigó la neurobiología de la mente y la conducta, con énfasis en las emociones. Este investigador demostró que en el cerebro las emociones y los pensamientos no son actividades separadas, sino que están interrelacionadas: "la emoción es el sistema sobre el que se apoya el proceso de construcción racional. Sin este soporte, no habría proceso posible."

Como plantea Mitchell (2009) especialista en pedagogía Waldorf, el docente tiene que tener presente que las habilidades socioemocionales de los niños los conducen a ellos a aprender a comunicarse, a conectarse con otros, resolver conflictos y afrontar desafíos. Estas habilidades les dan confianza para alcanzar metas, y la habilidad de hacer frente a las dificultades de la vida. En este sentido, desde esta pedagogía se estimula a los niños a desarrollar sus conductas prosociales. Investigaciones en alumnos graduados en las escuelas Waldorf han indicado que la currícula contenía el desarrollo de habilidades socioemocionales como una de las fortalezas del currículum (Mitchell, 2009).

La competencia social se entiende como la capacidad de conseguir los objetivos de una situación interpersonal, manteniendo una relación con el interlocutor a través del equilibrio de poder y de relaciones positivas (Del Prette \& Del Prette, 1999). A su vez, Tyler (1984) la define como el modo en que un individuo interacciona con los acontecimientos de su vida, tanto en el sentido de la resolución de problemas como de autorrealización. Según este planteo, un individuo competente es capaz de demostrar sus potencialidades y sentimientos positivos en relación a sí mismo, es decir, es capaz de establecer metas y trazar estrategias para conseguir buenos resultados incluso cuando fracasa (Cecconello, 1999). Esto es coherente con los objetivos que se plantea la pedagogía Waldorf en el desarrollo de las habilidades sociales del niño. El énfasis de dicha pedagogía consiste en educar y apoyar al niño en su desarrollo individual en un contexto comunitario. De esta forma se incentiva el desarrollo de todas las fuerzas y facultades del niño en crecimiento, estimulando el interés por el mundo, creatividad, pensamiento individual, la fuerza de voluntad, la inteligencia emocional y la competencia social (Carlgren, 2004). Se puede pensar que este peso que la escuela Waldorf pone en la competencia puede redundar en efectos 
sobre las habilidades de empatía, tal como se ve en otros estudios. Existen estudios que demuestran la relación, en mayor o menor grado, entre competencia social y empatía (Miville, Carlozzi, Gushue, Schara, \& Veda, 2006).

Kliewer (1991) indica que los niños más competentes socialmente son los más hábiles para comprender normas sociales, para interaccionar con sus pares y con los adultos y para regular sus emociones. La socialización de las emociones es un aspecto de central importancia para la expresividad emocional y la empatía (Eisenberg, Fabes \& Murphy, 1996). En este sentido, se podría decir que la pedagogía Waldorf estimula la competencia social. Un niño socialmente competente es capaz de ser sensible y empático con sus padres, de implicarse en actividades sociales positivas, de formar relaciones de amistad y de adaptarse a situaciones de estrés (Howes, Matheson \& Hamilton, 1994).

Golderos (2003) señaló que el proceso de aprendizajequeimplementala pedagogía Waldorf se afianza según avanzan las investigaciones en neurociencias. El método Waldorf pondera la armonía entre cuerpo y alma, haciendo hincapié en las sensaciones y emociones, lo cual tiene una importancia en el proceso de aprendizaje. Las escuelas Waldorf trabajan sobre estas bases desde hace más de ochenta años y realiza desde hace tiempo aquello que investigadores como LeDoux (2000) o Damasio (1996) describieron acerca de la relación entre emoción y cognición.

Mestre, Guil, Lopes, Salovey y Gil-Olarte (2006) explican que hay tres aspectos clave acerca de cómo las habilidades emocionales pueden contribuir a la adaptación social y académica del estudiante: 1. En primer lugar el trabajo académico y el desarrollo intelectual requiere utilizar emociones para facilitar el pensamiento, 2. En segundo lugar, la adaptación social en el ámbito educativo se relaciona con establecer relaciones de armonía con los pares y los maestros. En este sentido, las habilidades sociales juegan un papel crucial. 3. En tercer lugar, la adaptación social en la escuela y el rendimiento se pueden fortalecer mutuamente y además pueden estimular la motivación de los alumnos para implicarse en el trabajo diario y desarrollar mejores relaciones sociales con los compañeros.

Se puede concluir que las diferencias encontradas entre los alumnos de las distintas escuelas podrían deberse a los distintos modelos pedagógicos que recibieron. La escuela Waldorf hace énfasis en estimular la competencia social, por lo que esto pudo repercutir en las habilidades empáticas de los niños.

Como limitación del trabajo se puede indicar que tal vez haya otras variables que influyan sobre la empatía, como por ejemplo la personalidad de los niños evaluados. Para controlar esto, es deseable en un futuro estudio evaluarlos también en personalidad y aumentar las muestras. También podría ser recomendable evaluar estilos parentales y seguir realizando estudios sistemáticos en pedagogía Waldorf, para evaluar sus efectos sobre procesos básicos como inteligencia, inteligencia emocional y funciones ejecutivas. 


\section{Artículos Empíricos}

\section{Referencias}

Acevedo, J. A. \& Carrillo, M. L. (2010). Adaptación, Ansiedad y Autoestima en Niños de 9 a 12 años: una Comparación entre Escuela Tradicional y Montessori. Psicología Iberoamericana, 1, 19-29.

Barz, H., \& Randoll, D. (2007). Absolventen von Waldorfschulen. Eine Empirische Studie zu Bildung und Lebensgestatung Ehemaliger Waldodrfschueler.

Carlgren, F. (2004). Pedagogía Waldorf. Una educación hacia la libertad. Buenos Aires: Antroposofica.

Cencconello, A. M. (1999). Competência social, empatia e representacâo mental da relacâo de apego em familias em situacâo de risco. Tesis no publicada.

Damasio, A. R. (1996). El error de Descartes. Barcelona: Crítica.

Davis, M. H. (1980). A multidimensional Approach to inividual Differences in Empathy. Psychologhy, 10, 85- 97.

Del Prette, Z. A. P., \& Del Prette, A (1999). Psicología das habilidades sociais: terapia e educacao. Río de Janeiro: Vozes.

Eisenberg, N., Fabes, R. A., \& Murphy, B. C. (1996). Parent's reactions to children's negative emotions: relations to children's social competence and comforting behaviour. Child Development, 67, 2227-2247.

Golderos, M. A. (2003). Pedagogía Waldorf: el respeto al ser humano. Revista Aula, 119, 135-150.
Howes, C., Matheson, C.C., \& Hamilton, C. E. (1994). Maternal, teacher and child care history correlates of children's relationships with peers. Child Development, 65, 264273.

Kliewer, W. (1991). Doping in middle childhood: relations to competence, type A behaviour, monitoring, blunting, and locus of control. Developmental Psychology, 27, 689-697.

LeDoux, J. E. (2000). Emotion circuits in the brain. Annual Review of Neuroscience, 23, 155-184.

Mestre, V., Frias, M. D., \& Samper, P. (2004). La medida de la empatía: análisis del Interpersonal Reactivity Index. Psicothema, 16, 255-260.

Mestre, J. M., Guil, R., Lopes, P., Salovey, P., \& GilOlarte, P. (2006). Emotional intelligence and social and academic adaptation to school. Psicothema, 18, 112-117.

Mitchell, D. (2009). Social-Emotional education and Waldorf education. Research Bulletin, 2, 35-42.

Miville, M. L., Carlozzi, A.F., Gushue, G.V., Schara, S.L., \& Veda, M. (2006). Mental health counselor qualities for a diverse clientele: Linking empathy, universaldiverse orientation and emotional intelligence. Journal of Mental Health Counseling, 28, 151-165

Navarro, A. (2010). Tendencias pedagógicas contemporáneas. La pedagogía tradicional y el enfoque histórico-cultural. Análisis 


\section{Artículos Empíricos}

comparativo. Revista Cubana de Estomatología, 1, 12-27.

Palacios, J. (1985). Reflexiones en torno a las implicaciones educativas de la obra de Vigostki. Madrid: Sociedad Española de Psicología.

Palacios, J. (2010). La cuestión escolar. Buenos Aires: Ediciones Colihue

Petrash, J. (2009). Waldorf Education in Canada. Washigton: Nova Institute
Richaud, M. C. (2008). Evaluación de la empatía en población infantil argentina. IIPSI, 11, $101-115$.

Steiner, R. (1985). An Introduction to Waldorf Education. Berlin: Anthroposophic Press.

Tyler, F. (1984). El comportamiento psicosocial, la competencia psicosocial individual y las redes de intercambio de recursos como ejemplos de psicología comunitaria. Revista Latinoamericana de Psicología, 16, 77-92. 